\title{
AVALIAÇÃO DO CUIDADO AO USUÁRIO PORTADOR DE DIABETES EM UNIDADE DE SAÚDE
}

Aline Mariana Gafuri ${ }^{1}$, Beatris Mario Martinn', Carolina Passos Rocha ${ }^{1}$, Fernanda Rackes da Silva ${ }^{2}$, Roberta Luiza Longo', Guilherme Souza Cavalcanti de Albuquerque ${ }^{3}$

RESUMO: O presente trabalho teve como objetivo avaliar o cuidado programado ao diabético em Unidade Básica de Saúde do Sistema Único de Saúde de um município da região metropolitana de Curitiba, Paraná. Estudantes e professores de projeto de extensão identificaram os diabéticos cadastrados e analisaram o controle da doença pela unidade de saúde. Os usuários que não aderiam aos cuidados ou não obtinham melhora foram visitados para identificação dos motivos, orientação do cuidado e agendamento de consultas ou exames. Após o quarto mês, repetiu-se a análise, verificando-se expressiva melhora na adesão e no controle do diabetes. A partir disso, houve revisão do planejamento e avaliação do cuidado. A abertura do serviço para a participação da universidade constituiu importante espaço de aprendizagem e contribuiu para qualificar o atendimento aos usuários, objetivo maior dos serviços de saúde.

PALAVRAS-CHAVE: Diabetes mellitus; Centros de saúde; Sistema único de saúde.

\section{EVALUATION OF THE CARE GIVEN TO DIABETIC SERVICE USERS IN A HEALTH CARE UNIT}

ABSTRACT: This study aims to evaluate the care programmed for diabetics in a Basic Healthcare Unit of the Brazilian National Health in a county in the metropolitan region of Curitiba, in the state of Paraná. Students and professors of an extension project identified the diabetics registered in the unit and analysed how it controlled the condition. Service users who did not comply with treatment or who did not experience improvements in their condition were visited for identification of the reasons, care orientation and the booking of checkups or exams. After the fourth month the analysis was repeated, showing a significant improvement in compliance and in the control of the patients' diabetes. Based on this, there was a revision of the planning and evaluation of care. The opening of the unit to the university's participation constituted an important learning space and contributed to modifying the assistance given to service users, the main objective of the health services.

KEYWORDS: Diabetes mellitus; Center health; Unified health system.

\section{EVALUACIÓN DEL CUIDADO AL USUARIO PORTADOR DE DIABETIS EN UNIDAD DE SALUD}

RESUMEN: Este trabajo tuvo como objetivo evaluar el cuidado programado al diabético en Unidad Básica de Salud del Sistema Único de Salud de un municipio de la región metropolitana de Curitiba, Paraná. Estudiantes y profesores de proyecto de extensión identificaron los diabéticos registrados y analizaron el control de la enfermedad por la unidad de salud. Los usuarios que no participaron de los cuidados o no mejoraron fueron visitados para identificación de los motivos, orientación del cuidado y para concertar consultas o exámenes. Despues del cuarto mes, se repitió el análisis, verificándose expresiva mejora en la adhesión y en el control del diabetis. Así, hubo revisión del planeamiento y evaluación del cuidado. La apertura del servicio para la participación de la universidad constituyó importante espacio de aprendizaje y contribuyó para cualificar el atendimiento a los usuarios, objetivo principal de los servicios de salud.

PALABRAS-CLAVE: Diabetis mellitus; Unidad básica de salud; Sistema único de salud.

${ }^{1}$ Acadêmico de Medicina da Universidade Federal do Paraná - UFPR.

${ }^{2}$ Acadêmica de Enfermagem da UFPR.

${ }^{3}$ Médico. Doutor em Educação. Professor do Departamento de Saúde Comunitária da UFPR. 


\section{INTRODUÇÃO}

O Diabetes mellitus (DM) constitui um conjunto de disfunções metabólicas caracterizado principalmente por hiperglicemia, associado a alterações nos níveis de insulina ${ }^{(1-2)}$. Em âmbito mundial, sabe-se que, em 2007, atingiu cerca de 7\% da população total, sendo que a maior parte dos casos encontra-se em países desenvolvidos ${ }^{(3)}$. No Brasil, a prevalência estimada é de $11 \%$ da população com idade igual ou superior a 40 anos, o que, em números absolutos, constituem 5,5 milhões de pessoas ${ }^{(3)}$. O impacto dessa doença nos gastos públicos nacionais é relativamente grande e varia de $2,5 \%$ a $15 \%$ dos gastos em saúde ${ }^{(4)}$. Estima-se que o indivíduo portador de DM apresente um custo de hospitalização duas a quatro vezes maior que os não diabéticos. O custo social da doença também é bastante considerável, uma vez que as complicações crônicas do DM são as principais responsáveis pela morbidade e mortalidade dos indivíduos com a doença ${ }^{(5)}$.

Os portadores do DM podem desenvolver inúmeras complicações, dentre as quais insuficiência renal, cegueira, doenças cardiovasculares e alterações vasculares que podem exigir a amputação de membros, em casos de pé diabético, em especial quando não devidamente monitorados, ou quando não se obtém o controle glicêmico. Para a prevenção da enfermidade e das consequências citadas, a ação de saúde deveria estar voltada para a mudança do modo de vida dos diversos grupos sociais, considerando-se o modo de vida como aquele relativo a processos particulares de grupos populacionais característicos, e que marcam traços específicos de diferentes grupos sociais, delimitando processos singulares ${ }^{(6)} . \mathrm{Ou}$ seja, as ações de saúde, em associação necessária com outros âmbitos da vida em sociedade (economia, política, infraestrutura, cultura) devem resultar em mudanças mais gerais, que por sua vez permitirão modos de vida mais saudáveis. A partir disso, a assistência à saúde pode agir mesmo que nos limites estreitos da ação centrada no indivíduo, com a instituição do tratamento adequado e de cuidado continuado, auxiliando o usuário a melhorar alguns aspectos do seu estilo de vida. São exemplos disso a adoção de uma dieta saudável, atividades físicas, exame diário e cuidados adequados dos pés, além do uso correto da medicação. Desta forma, as complicações podem ser minimizadas ou retardadas, obtendo-se, além da melhora da qualidade de vida, a redução dos custos da assistência médica ${ }^{(1,7)}$.

O diagnóstico, o cuidado e o acompanhamento destes indivíduos são hoje prioridades do Ministério da Saúde ${ }^{(1)}$.
Na atenção básica, estas ações organizam-se em três níveis de prevenção: o primeiro trata de prevenção dos fatores de risco, o segundo de diagnóstico de novos doentes e o terceiro de monitoramento dos já diagnosticados. Nesta perspectiva, foi instituído pelo Ministério da Saúde o Programa "Hiperdia", que atende à prevenção geral do diabetes e da hipertensão. Este programa visa o cadastramento e o acompanhamento dos doentes, bem como o planejamento de estratégias de saúde pública que resultem em uma melhoria do quadro atual dessas doenças.

No presente estudo, analisou-se o cuidado aos diabéticos no Programa "Hiperdia" realizado por uma equipe da Estratégia Saúde da Família do Município de Colombo - PR e, a partir dos resultados encontrados, implantaram-se medidas para a adequação das ações do programa e a melhoria do controle do diabetes na região.

\section{METODOLOGIA}

A pesquisa foi realizada em uma Unidade Básica de Saúde (UBS) de Colombo-Paraná, por acadêmicos de Medicina e Enfermagem da Universidade Federal do Paraná(UFPR). Colombo é um município situado na região metropolitana de Curitiba, com uma população aproximada de 220 mil habitantes; possui vinte e três unidades de saúde, sendo que em vinte delas o Programa "Hiperdia" está implantado. A UBS em questão foi escolhida por constituir campo de extensão universitária para acadêmicos de Medicina, Enfermagem e Nutrição, bem como para integrantes da Residência Multiprofissional em Saúde da Família.

A pesquisa envolveu a população da área identificada como "112", atendida por uma das três equipes de Saúde da Família da UBS, composta por um médico, uma enfermeira, cinco agentes comunitários de saúde e uma auxiliar de enfermagem.

O presente estudo foi analisado e aprovado pelo Comitê de Ética do Setor de Ciências da Saúde da UFPR, com registro CEP/SD 798.133.09.09. O estudo caracteriza-se por uma abordagem quantitativa e descritiva, utilizando como fonte para o levantamento de dados todos os prontuários referentes a usuários moradores da área "112". A análise de dados foi realizada de modo a comparar os resultados obtidos antes e depois da intervenção dos acadêmicos.

Inicialmente, organizou-se uma lista de usuários cadastrados no Programa "Hiperdia". A partir desta lista, procedeu-se a análise dos dados dos prontuários e, com o acompanhamento dos agentes comunitários de saúde da equipe responsável pela área, localizou-se 
cada usuário em seu domicílio. Todos foram previamente informados sobre a pesquisa e somente participaram da mesma após expressar sua concordância mediante assinatura do Termo de Consentimento Livre e Esclarecido.

No período de abril a julho de 2008 realizaram-se visitas domiciliares a todos os indivíduos localizados, indagando-os quanto à alimentação, o controle da pressão arterial, o uso de medicamentos, exames laboratoriais realizados, a data da última consulta e sobre complicações do diabetes; também foram solicitadas informações acerca da participação e acompanhamento da doença junto à UBS. Aqueles usuários que não possuíam resultados de exames ou que não haviam comparecido a consultas médicas no tempo preconizado pelo programa foram devidamente encaminhados para a Unidade de Saúde.

Neste estudo, 79 indivíduos compuseram o universo pesquisado, pois das 85 pessoas identificadas, seis não tiveram sua moradia localizada, ou estavam sempre ausentes nos horários das visitas domiciliares.

Após estas ações iniciais, procedeu-se a uma nova análise dos prontuários, entre os meses de agosto e outubro do mesmo ano, buscando comparar os resultados quanto ao número de presenças dos usuários às atividades do programa, à realização dos exames complementares e ao controle dos níveis glicêmicos. Verificou-se a significância através do cálculo dos valores de $p$ a partir do teste qui quadrado. Com isso, pretendeu-se ressaltar a eficácia da ação desenvolvida pela equipe da UBS, com o apoio dos pesquisadores.

\section{RESULTADOS}

A UBS na qual foi realizado este estudo abrange uma população de 13.000 habitantes, dos quais 4.312 pertencem à área estudada. Até abril de 2008, dos 132 usuários portadores de DM estimados para a população da área, de acordo com os critérios do Ministério da Saúde ${ }^{(1)}$, a equipe da UBS havia identificado $85(64,4 \%)$, sendo que somente 67 destes, ou seja, $78,8 \%$ dos identificados (50,7\% do total de portadores estimados), estavam inscritos no programa e 41 ( $61 \%$ dos inscritos ou $31,06 \%$ do total de estimados), participavam efetivamente do mesmo (Gráfico 1).

Dentre os 67 inscritos no "Hiperdia", 38, ou seja, 56,7\% ( $28,8 \%$ do total estimados) possuíam o resultado da glicemia de jejum nos últimos seis meses ( $\mathrm{G}<6 \mathrm{~m})$ e destes, somente $16,42,1 \%$ (12,1\% do total de diabéticos estimados) apresentavam valores abaixo de $120 \mathrm{mg} / \mathrm{dL}$ (G controlada). Em relação à hemoglobina glicada, apenas 10 , ou $14,9 \%$ dos inscritos no programa $(7,63 \%$ do total de diabéticos estimados) apresentavam resultados referentes aos últimos seis meses e, entre estes, 33,3\% (2,3\% do total de diabéticos estimados) apresentavam valores iguais ou abaixo de $7 \%$. Ao final de outubro de 2008, com a ajuda dos pesquisadores, três novos indivíduos foram identificados como portadores de DM, e a UBS passou a contar com 88 pacientes identificados. Com isso, houve uma leve melhora no percentual dos identificados, passando de $64,3 \%$ para $66,7 \%$ dos 132 portadores de

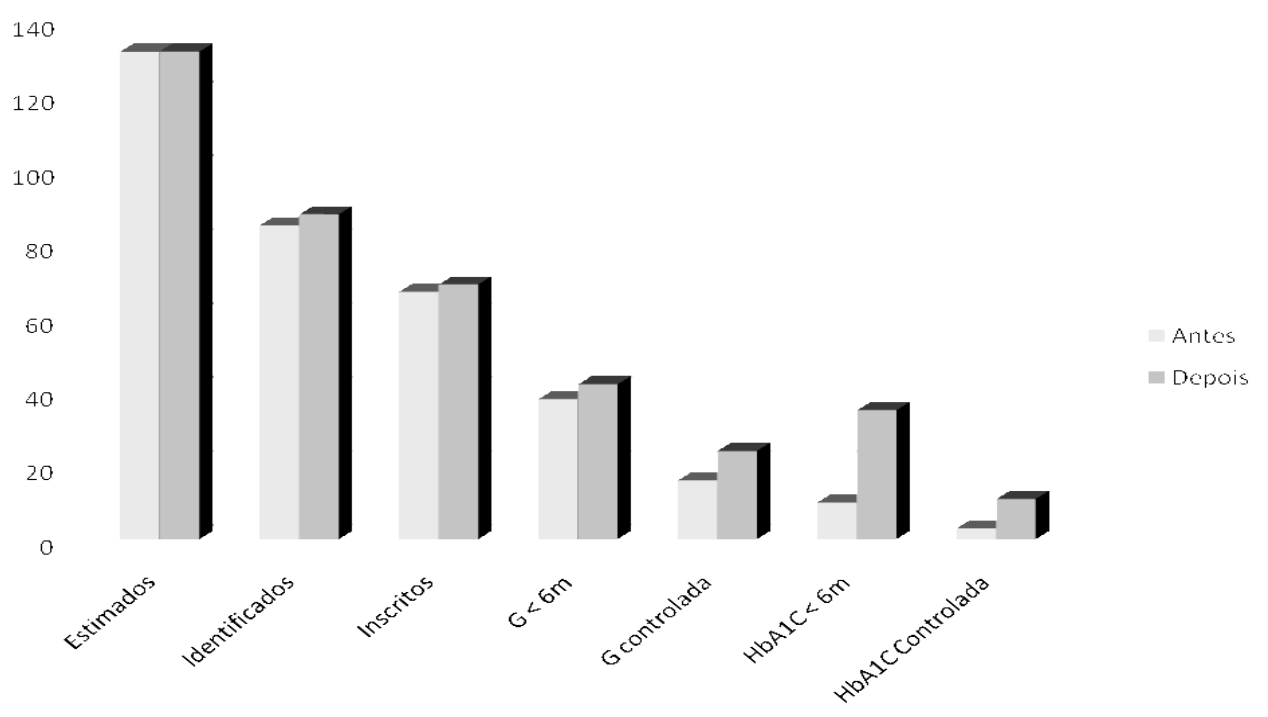

Gráfico 1 - Comparação dos usuários diabéticos, demonstrando o número de estimados, identificados, inscritos no "Hiperdia", exames de glicemia de jejum e hemoglobina glicada realizados antes e depois da atuação dos pesquisadores. Colombo, 2009 
DM estimados para a área "112". Dos identificados, $69(78,4 \%)$ estavam inscritos no "Hiperdia". Entre os usuários cadastrados no programa, 42 , ou $60,87 \%$ (31,8\% dos 132 estimados para a área) haviam realizado o exame de glicemia de jejum nos últimos seis meses e 35 ou $50,7 \%$ (26,5\% dos 132 estimados para a área) tinham feito o exame de hemoglobina glicada para o mesmo período.

No que diz respeito ao controle dos níveis glicêmicos, após a intervenção, $31,4 \%$ dos pacientes que realizaram ao exame de hemoglobina glicada apresentavam resultados considerados normais, contra $33,3 \%$ antes da intervenção. Isto se deve ao fato de que, no primeiro momento, apenas 10 pacientes haviam realizado o exame, três dos quais estavam dentro dos limites da normalidade. Quanto à glicemia, após a intervenção, 57,1\% dos pacientes que haviam realizado o exame encontravam-se com níveis considerados normais, contra $42,1 \%$ antes da intervenção (Tabela 1).

Tabela 1 - Comparação dos resultados dos exames de controle do diabetes antes e depois da intervenção na UBS. Colombo, 2009

\begin{tabular}{lcc}
\hline \multicolumn{1}{c}{ Exames } & $\begin{array}{c}\text { Antes da in- } \\
\text { tervenção dos } \\
\text { acadêmicos (\%) }\end{array}$ & $\begin{array}{c}\text { Depois da } \\
\text { intervenção dos } \\
\text { acadêmicos (\%) }\end{array}$ \\
\hline $\begin{array}{l}\text { Glicemia em } \\
\text { jejum }\end{array}$ & $56,7 \%$ & $60,87 \%$ \\
$\begin{array}{l}\text { Hemoglobina } \\
\text { glicada }\end{array}$ & $14,9 \%$ & $50,7 \%$ \\
$\begin{array}{l}\text { Glicemia } \\
<120 \mathrm{mg} / \mathrm{dl}\end{array}$ & $42,1 \%$ & $57,1 \%$ \\
$\begin{array}{l}\text { Hemoglobina } \\
\text { glicada }<7 \%\end{array}$ & $33,3 \%$ & $31,4 \%$ \\
\hline
\end{tabular}

\section{DISCUSSÃO}

De acordo com documento da Sociedade Brasileira de Diabetes ${ }^{(8)}$, os dados estadunidenses utilizados na avaliação dos principais parâmetros de qualidade de assistência médica são de $72 \%$ dos portadores com exames de hemoglobina glicada, sendo que $39 \%$ apresentam valores acima de 9,5\% (alto risco). Ainda que considerados precários pela Sociedade Brasileira de Diabetes, estes valores são próximos aos encontrados na área "112" da UBS de Colombo, mesmo após as mudanças instituídas com o presente trabalho. Estudo realizado em PelotasRS demonstrou que todos os médicos relatam solicitar exames de glicemia de jejum, enquanto apenas $60 \%$ dos médicos dizem pedir o exame de hemoglobina glicada no acompanhamento do indivíduo diabético ${ }^{(9)}$.

$\mathrm{O}$ controle obtido pelas pessoas acompanhadas no Hospital de Clínicas de Ribeirão Preto-SP foi de 28,8\% da amostra com dados de glicemia abaixo de $110 \mathrm{mg} / \mathrm{dl}$, e $21,2 \%$ com dados de hemoglobina glicada abaixo de $8 \%$. O mesmo estudo aponta para uma baixa adesão aos programas educativos: de uma média de 350 pacientes por mês, apenas 20 participam do grupo de educação ${ }^{(10)}$. A baixa adesão a este tipo de atividade também foi verificada em Colombo, uma vez que dos cadastrados no programa somente cerca de $60 \%$, como registrado acima, participavam das reuniões do "Hiperdia".

Os valores de referência para hemoglobina glicada variam conforme o consenso da época de realização de cada estudo, de acordo com as evidências o estabelecimento de valores mais baixos reduz as chances de danos decorrentes do diabetes. No presente estudo, utilizamos como valor normal $7 \%{ }^{(11)}$, que, embora seja diferente do utilizado nos estudos dos EUA (9,5\%) e de Ribeirão Preto (8\%), acima citados ${ }^{(8,10)}$, aponta, da mesma forma, para grande dificuldade no controle glicêmico.

A diferença existente entre o número de portadores de DM identificados e os cadastrados, pelo que se pode levantar junto aos indivíduos no presente estudo, deve-se, principalmente, ao horário de realização das atividades (consultas e reuniões), que coincide com o horário de trabalho de uma parcela significativa destas pessoas; e, em segundo lugar, à preferência de alguns usuários por acompanhamentos em clínicas privadas e hospitais de grande porte.

Os estilos de vida, bem como as expressões genofenotípicas dos indivíduos, ocorrem dentro dos limites dados pelas condições de existência, o modo de vida dos diversos grupos sociais, este sendo determinado pelas leis mais gerais de organização da sociedade $e^{(6,11-13)}$.

Conclui-se que os dados encontrados apontam para uma melhora do acompanhamento ao usuário diabético quando comparados com os dados iniciais. Percebe-se, também, uma melhora no controle dos níveis glicêmicos ao compararmos os resultados dos exames. No entanto, destes resultados, o único que mostrou diferença estatisticamente significativa foi a proporção de diabéticos que realizaram exame de hemoglobina glicada nos últimos seis meses. A diferença de proporção entre diabéticos com glicemia normal, antes e depois da intervenção, apesar de apresentar proporções mais elevadas depois da intervenção, não se mostrou estatisticamente significativa. Há limitações, contudo, que podem interferir 
na análise, devido ao reduzido tamanho da população estudada e ao pequeno tempo de acompanhamento. De qualquer forma, todos os resultados apontam para uma possibilidade de mudanças animadoras.

\section{CONSIDERAÇÕES FINAIS}

A partir dos dados referentes à DM observados antes e depois da presença dos acadêmicos observa-se que o trabalho foi gerador de mudanças, tanto pela atuação direta na comunidade como por centrar mais a atenção da equipe da UBS no impacto do cuidado ao paciente diabético. A unidade foi capaz de identificar de forma mais clara os diabéticos não-participantes e melhorar o controle da glicemia dos pacientes sob seu cuidado.

Além disso, percebe-se que mesmo entre os participantes do "Hiperdia" que aderiam ao programa de cuidados, o controle da glicemia não chegava a $60 \%$, demonstrando que a determinação da condição estudada não se limita ao vínculo com a unidade ou à participação nas reuniões do "Hiperdia" e que a determinação do adoecimento e do controle da doença ultrapassam os limites das condições singulares de existência. O livre arbítrio não é assim tão livre que permita a cada um levar uma vida saudável, na dependência, apenas, do esforço individual. Centrar o cuidado à saúde em tal pressuposto constitui, neste sentido, um erro de origem dos serviços de saúde.

\section{REFERÊNCIAS}

1. Ministério da Saúde (BR). Secretaria de Atenção à Saúde. Departamento de Atenção Básica. Cadernos de atenção básica. Diabetes mellitus. Protocolo. Brasília: Ministério da Sáude. 2006.

2. Lopes AC. Tratado de clínica médica. São Paulo: Roca, 2006. V. 2

3. Ministério da Saúde(BR) [Internet]. Brasília: MS; [Acesso em 04 mar 2009]. Informe da Atenção Básica. Disponível: http://http://dab.saude.gov.br/

4. McLellan KCP, Motta DG, Lerario AC, Campino ACC. Custo do atendimento ambulatorial e gasto hospitalar do diabetes mellitus tipo 2. Saude Rev. 2006;8(20):37-45.

5. Gross JL, Nehme M. Detecção e tratamento das complicações crônicas do diabetes melito: consenso da Sociedade Brasileira de Diabetes e Conselho Brasileiro de Oftalmologia. Rev Assoc Med Bras. 1999;45(3):279-84.
6. Breilh, J. Nuevos conceptos y técnicas de investigación: guia pedagógica para un taller de metodologia. $3^{\mathrm{a}}$ ed. Quito: CEAS, 1997.

7. Carvalho RDP, Carvalho CDP, Martins DA. Aplicação dos cuidados com os pés entre portadores de diabetes mellitus. Cogitare Enferm. 2010;15(1):106-9.

8. Sociedade Brasileira de Diabetes. [Internet]. Proposta preliminar para avaliação de viabilidade de implementação pela Sociedade Brasileira de Diabetes. [Acesso em 04 mar 2009]. Disponível: http://www. diabetes.org.br/protocolos/cqsbd.php.

9. Assunção MCF. Atenção primária em diabetes no sul do Brasil: estrutura, processo e resultado. Rev Saude Publica. 2001;35(1):88-95.

10. Cazarini RP, Zanetti ML, Ribeiro KP, Pace AE, Foss MC. Adesão a um grupo educativo de pessoas portadoras de diabetes mellitus: porcentagem e causas. Medicina. 2002;35;142-50.

11. Ducan BB, Schmidt MI, Giugliani ERJ. Medicina ambulatorial: condutas de atenção primária baseadas em evidências. $3^{\mathrm{a}}$ ed. Porto Alegre: Artmed, 2006.

12. Breilh J, Granda E. Saúde na sociedade: guia pedagógico sobre um novo enfoque do método epidemiológico. $2^{\mathrm{a}}$ ed. São Paulo: Cortez; Rio de Janeiro: ABRASCO, 1989.

13. Breilh J. Epidemiologia crítica: ciência emancipadora e interculturalidade. Rio de Janeiro: Fiocruz, 2006. 